\title{
ORIGINAL ARTICLE Positron emission tomography-computed tomography in the diagnostic evaluation of smoldering multiple myeloma: identification of patients needing therapy
}

\author{
B Siontis ${ }^{1}$, S Kumar ${ }^{2}$, A Dispenzieri ${ }^{2}$, MT Drake ${ }^{3}$, MQ Lacy ${ }^{2}$, F Buadi $^{2}$, D Dingli ${ }^{2}$, P Kapoor ${ }^{2}$, W Gonsalves ${ }^{2}$, MA Gertz ${ }^{2}$ and SV Rajkumar ${ }^{2}$
}

We studied 188 patients with a suspected smoldering multiple myeloma (MM) who had undergone a positron emission tomography-computed tomography (PET-CT) scan as part of their clinical evaluation. PET-CT was positive (clinical radiologist interpretation of increased bone uptake and/or evidence of lytic bone destruction) in 74 patients and negative in 114 patients. Of these, 25 patients with a positive PET-CT and 97 patients with a negative PET-CT were observed without therapy and formed the study cohort $(n=122)$. The probability of progression to MM within 2 years was $75 \%$ in patients with a positive PET-CT observed without therapy compared with $30 \%$ in patients with a negative PET-CT; median time to progression was 21 months versus 60 months, respectively, $P=0.0008$. Of 25 patients with a positive PET-CT, the probability of progression was $87 \%$ at 2 years in those with evidence of underlying osteolysis $(n=16)$ and $61 \%$ in patients with abnormal PET-CT uptake but no evidence of osteolysis $(n=9)$. Patients with positive PET-CT and evidence of underlying osteolysis have a high risk of progression to MM within 2 years when observed without therapy. These observations support recent changes to imaging requirements in the International Myeloma Working Group updated diagnostic criteria for MM.

Blood Cancer Journal (2015) 5, e364; doi:10.1038/bcj.2015.87; published online 23 October 2015

\section{INTRODUCTION}

Smoldering multiple myeloma (SMM) is an intermediate stage between monoclonal gammopathy of undetermined significance and active $\mathrm{MM} .^{1}$ The risk of progression to active malignancy in the first 5 years of diagnosis is $~ 50 \%{ }^{2}$ According to International Myeloma Working Group (IMWG) criteria, the diagnosis of SMM requires clonal bone marrow plasma cells (BMPC) of 10-60\% and/ or a serum monoclonal $(\mathrm{M})$ protein $>3 \mathrm{~g} / \mathrm{dl}$, plus the absence of hypercalcemia, renal failure, anemia and bony lesions (CRAB features) or other myeloma defining events. ${ }^{3}$ The current standard of care is observation without therapy until development of symptoms ${ }^{4}$. Early treatment as opposed to watchful waiting of patients with highest risk of progression has the potential to improve progression-free and overall survival. ${ }^{5}$ The development of biomarkers to aid in distinguishing these high-risk patients is an area of active interest, and has included assessment of BMPC percentage, serum $M$ protein, serum-free light-chain ratio and immunophenotyping of aberrant plasma cells. ${ }^{2,6-10}$

Recently, interest in the use of imaging modalities other than bone surveys to risk stratify patients with SMM has emerged. Hillengass et al. ${ }^{11}$ found that the presence of one or more focal lesion on magnetic resonance imaging in patients with SMM was a strong predictor of progression to active MM. They also found that progression on magnetic resonance imaging was associated with a high probability of progression to $\mathrm{MM}$, regardless of the initial magnetic resonance imaging finding. ${ }^{12}$

Limited data are available on the use of 18F-fluorodeoxyglucose positron emission tomography combined with computerized tomography (PET-CT) to guide decision making in SMM. We hypothesize that PET-CT imaging at the time of SMM diagnosis can be used to identify patients at high risk of disease progression within 2 years.

\section{MATERIALS AND METHODS}

We identified all patients with a diagnosis of SMM (based on standard disease definition prior to recent updated IMWG criteria) from January 2000 to March 2014 who had undergone a PET-CT scan as part of their clinical evaluation by using the Mayo Clinic Data Discovery and Query Database and a review of available medical records. The PET-CT findings, results of other diagnostic tests and clinical course were then abstracted. A positive PET-CT was defined as a radiologist interpretation of abnormal increased uptake (diffuse and/or one or more focal skeletal areas) and/or evidence of lytic bone destruction on the CT portion of the exam. The primary end point was progression to active MM within the first 2 years following a positive PET-CT result among patients observed without therapy. Secondary end points included the proportion of patients in whom the diagnosis of active MM was made based solely on the findings of the PET-CT, the probability of progression within 2 years in patients with a negative PET-CT who were observed without therapy, and estimating differences in the probability of progression based on the presence or absence of underlying osteolysis in patients with a positive PET-CT.

The $x^{2}$ test was used to compare nominal values. Time to progression (TTP) was measured from the date of PET-CT until progression to active MM. Kaplan-Meier analysis was performed to generate progression and survival curves. Time to event and survival between groups was compared with the two-tailed log-rank test.

\section{RESULTS}

One hundred and eighty-eight patients were identified with a suspected diagnosis of SMM in whom a PET-CT scan had been

\footnotetext{
'Department of Internal Medicine, Mayo Clinic, Rochester, MN, USA; ${ }^{2}$ Division of Hematology, Mayo Clinic, Rochester, MN, USA and ${ }^{3}$ Division of Endocrinology, Mayo Clinic, Rochester, MN, USA. Correspondence: Dr SV Rajkumar, Division of Hematology, Mayo Clinic, 200 First Stret SW, Rochester, MN 55905, USA. 


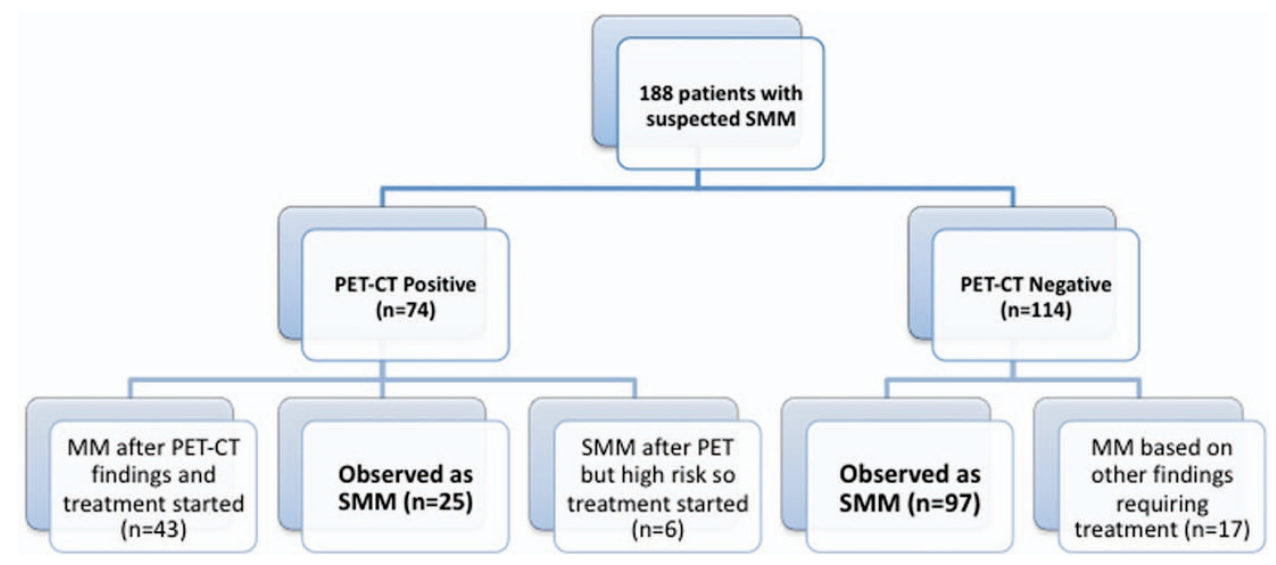

Figure 1. Disposition of patients. PET-CT, positron emission tomography-computerized tomography; SMM, smoldering multiple myeloma.

\begin{tabular}{|c|c|}
\hline & $\begin{array}{l}\text { Patients, } \\
\mathrm{N}=122\end{array}$ \\
\hline Median age, years (range) & $69(35-92)$ \\
\hline Female sex, $N(\%)$ & $61(50 \%)$ \\
\hline Serum monoclonal protein spike, median (range) $(\mathrm{g} / \mathrm{dl})$ & $2.0(0.0-4.6)$ \\
\hline \multicolumn{2}{|l|}{ Urine monoclonal protein spike $(\mathrm{n}=96), \mathrm{N}(\%)$} \\
\hline Not present & $29(30)$ \\
\hline Detected on immunofixation only & $39(41)$ \\
\hline Measurable but $<0.5 \mathrm{~g}$ per $24 \mathrm{~h}$ & $24(25)$ \\
\hline $0.5 \mathrm{~g}$ per $24 \mathrm{~h}$ or more & $3(3)$ \\
\hline Bone marrow plasma cell percentage, median (range) & $20(4.0-100)$ \\
\hline \multicolumn{2}{|l|}{ Serum-free light-chain assay $(n=91), N(\%)$} \\
\hline Abnormal $\kappa / \lambda$ FLC ratio $(<0.26$ or $>1.65)$ & $12(13)$ \\
\hline Abnormal involved/uninvolved FLC ratio $\geqslant 8$ & $57(63)$ \\
\hline Abnormal involved/uninvolved FLC ratio $\geqslant 100$ & $13(14)$ \\
\hline
\end{tabular}

performed as part of the diagnostic evaluation. PET-CT was positive in 74 patients, and negative in 114 patients. Of the 74 patients with a positive PET-CT, 49 were diagnosed and treated as MM, whereas 25 were considered to still have SMM and observed (Figure 1). Of the 49 patients diagnosed as MM, 12 (24\%) were upstaged to the diagnosis of active disease solely based on the findings of the PET-CT; in the remaining 37 patients, myeloma defining events were identified on other laboratory tests conducted during the same visit. Similarly, of the 114 patients with a negative PET-CT, 17 (14\%) were diagnosed and treated as MM based on other laboratory parameters, whereas 97 were considered to have SMM and observed. Thus, 25 patients with a positive PET-CT and 97 patients with a negative PET-CT who were observed without therapy formed the principal cohort $(n=122)$ for this study (Figure 1). Patient characteristics are provided in Table 1. The rate of progression to MM within 2 years was then compared between these two groups.

The probability of progression to MM within 2 years was $75 \%$ in patients with a positive PET-CT $(n=25)$ compared with $30 \%$ in patients with a negative PET-CT $(n=97)$; median TTP was 21 months versus 60 months, respectively, $P=0.0008$ (Figure 2). The mode of progression in patients with a positive PET-CT who progressed was anemia (10 patients, including one patient who also developed amyloidosis and one patient with central nervous system disease), bone disease (6 patients, including 2 patients

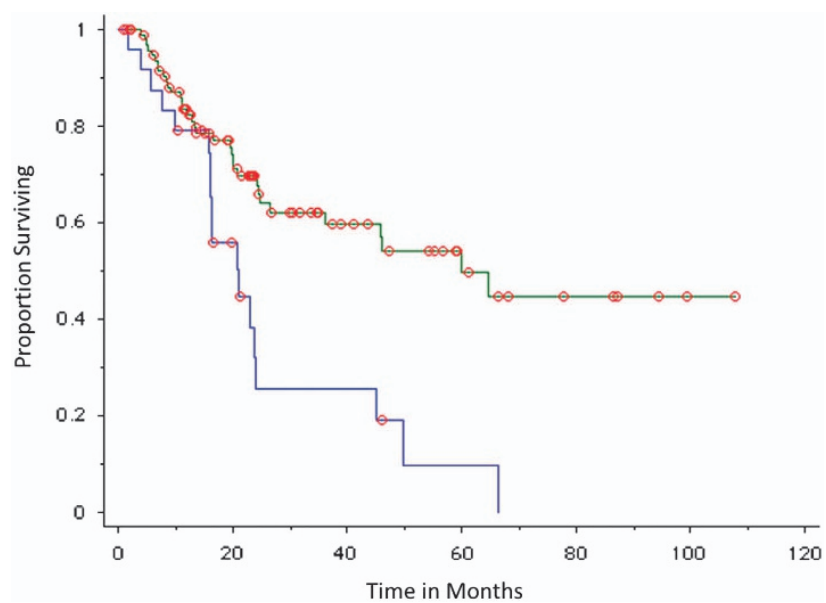

Figure 2. TTP of SMM in patients observed without therapy, including all patients with PET-CT imaging. Blue curves represent positive (abnormal PET-CT) imaging result; green curves represent negative imaging results.

with concurrent renal failure and one with cryoglobulinemia), renal failure ( 1 patient) and rapid rise in $M$ protein ( 1 patient).

The median TTP in patients with positive PET-CT and underlying osteolysis $(n=16)$ was 21 months compared with 60 months in patients with no evidence of osteolysis on PET-CT $(n=106)$, $P=0.004$ (Figure 3 ). Among patients with a positive PET-CT, the probability of progression was $87 \%$ at 2 years in the subset of patients with underlying osteolysis $(n=16)$ and $61 \%$ in patients with abnormal PET-CT uptake but no evidence of osteolysis $(n=9)$, $P=0.31$.

Analysis was then restricted to 59 patients in whom the PET-CT was carried out within 90 days of diagnosis of SMM. The probability of progression to MM within 2 years was $82 \%$ in patients with a positive PET-CT $(n=13)$ compared with $28 \%$ in patients with a negative PET-CT $(n=46)$; median TTP in patients with a positive PET-CT was 21 months compared with 65 months in patients with a negative PET-CT, $P=0.0006$ (Figure 4).

Median overall survival was not reached. The 5 -year survival rate was $61 \%$ versus $82 \%$ in patients with positive and negative PET$\mathrm{CT}$, respectively, but the difference was not statistically significant, $P=0.42$ (Figure 5).

Thirteen patients had a serum FLC ratio of $\geqslant 100$; of these, only three had a positive PET-CT and two had disease progression (both at 16 months). Five patients had a BMPC $\geqslant 60 \%$; none of these patients had a positive PET-CT. Only one patient had both 


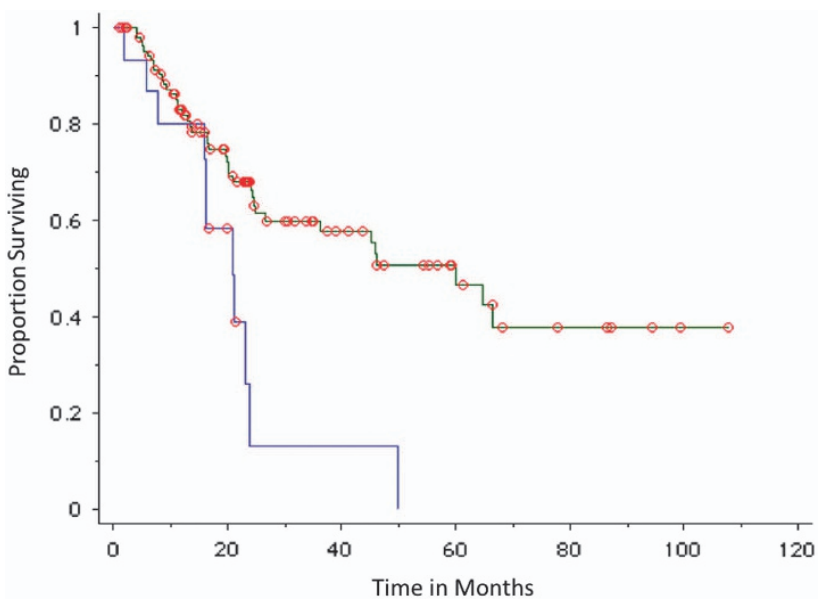

Figure 3. TTP of SMM in patients observed without therapy based on osteolysis on PET-CT. Blue curves represent positive (abnormal bone/osteolytic lesion present) imaging result; green curves represent negative (no bone abnormality) imaging results.

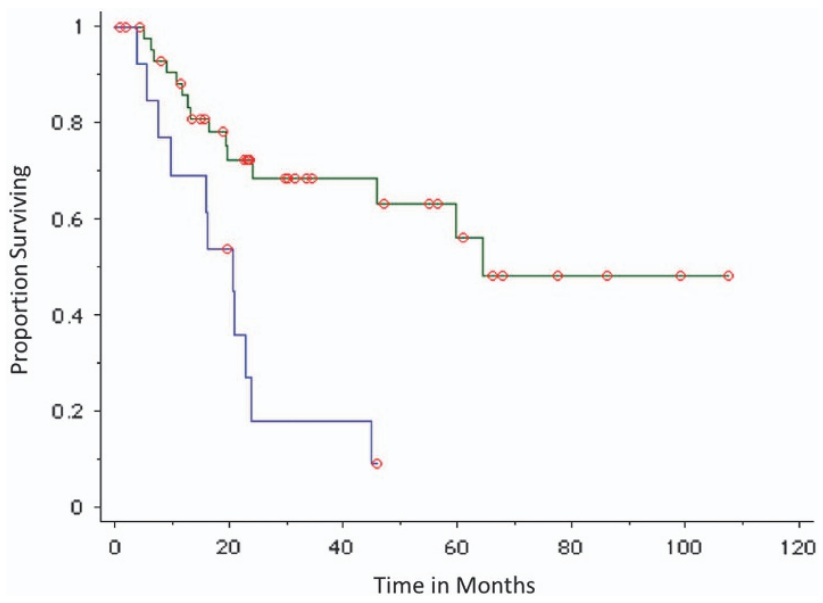

Figure 4. TTP of SMM in patients observed without therapy based on PET-CT imaging carried out within 90 days of diagnosis. Blue curves represent positive (abnormal PET-CT) imaging result; green curves represent negative imaging results.

serum FLC ratio of $\geqslant 100$ and BMPC $\geqslant 60 \%$. The probability of progression to $\mathrm{MM}$ in patients with a positive PET-CT remained unchanged when analysis was repeated after excluding the 17 patients who had either serum FLC ratio of $\geqslant 100$ or BMPC $\geqslant 60 \%$. The probability of progression to MM within 2 years was $77 \%$ in patients with a positive PET-CT $(n=22)$ compared with $31 \%$ in patients with a negative PET-CT $(n=83)$; median TTP was 21 months versus not reached, respectively, $P=0.0002$.

\section{DISCUSSION}

SMM is a plasma cell proliferative disorder with a risk of progression to MM of $\sim 10 \%$ per year in the first 5 years following diagnosis, 3\% per year subsequently for the next 5 years and $\sim 1.5 \%$ per year thereafter. ${ }^{2}$ While the current standard of care remains observation, concern exists that patients at high risk of progression can sustain unacceptable end-organ damage despite careful observation. ${ }^{13}$ Based on specific biomarkers, the IMWG recently reclassified as $\mathrm{MM}$ a small proportion of patients with SMM and ultra-high risk of progression ( 40\% per year in the first

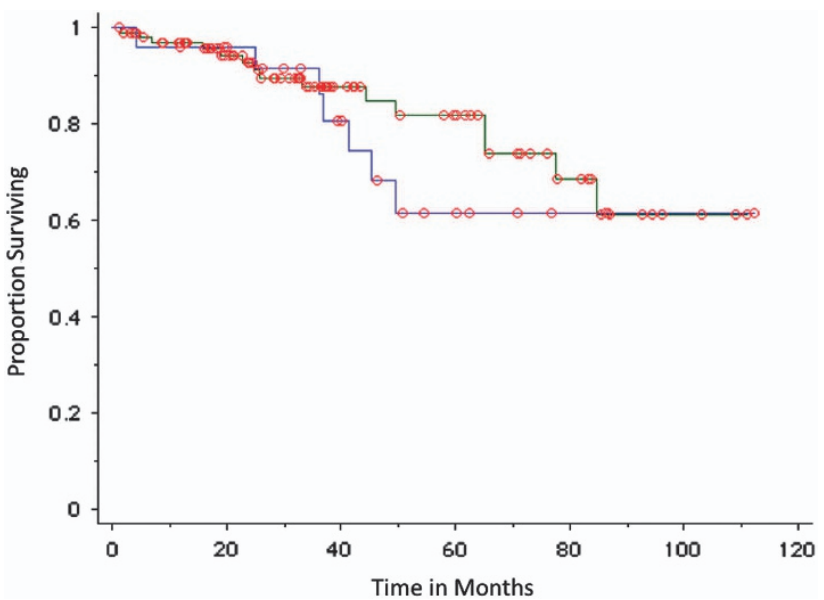

Figure 5. Overall survival of SMM patients based on PET-CT imaging results. Blue curve represents positive (abnormal) imaging results; green curve represents negative imaging results.

2 years). ${ }^{3}$ The IMWG also clarified that evidence of osteolysis on CT or PET-CT meets the definition of bone disease in MM. This recommendation was supported by a systematic review that compared newer imaging modalities, magnetic resonance imaging, 18F-fluorodeoxyglucose positron emission tomography, PET-CT and whole-body CT, to conventional whole-body skeletal radiography ${ }^{14}$ and found that newer imaging techniques had greater sensitivity compared with radiographic bone survey for the detection of MM bone lesions.

In clinical practice, patients with osteolytic lesions visible on CT or PET-CT but not seen on skeletal radiography have often been treated as MM even before the updated IMWG criteria were published. Coupled with the relative rarity of SMM, studies of the natural history of such patients based on the results of PET-CT imaging is scarce. In this study, we show that patients with suspected SMM who have an abnormal PET-CT (clinical radiologist interpretation of increased bone uptake and/or evidence of lytic bone destruction) who are observed without therapy are at high risk (75\%) of progression to MM within 2 years. This risk increases to $87 \%$ in patients with evidence of underlying osteolysis on PET-CT. Importantly, these estimates likely underestimate the true risk of progression to myeloma as they exclude patients with presumably higher grade lesions on PET-CT who were initiated on therapy based solely on the PET-CT finding ( $n=12$ in this cohort).

We found PET-CT identifies patients at high risk of progression independent of the new serum FLC and BMPC thresholds established to define MM in the revised IMWG criteria. Only three patients with those ultra-high-risk biomarkers (serum FLC ratio $\geqslant 100$ or BMPC $\geqslant 60 \%$ ) had a positive PET-CT in our study. The probability of progression to MM was not affected when patients meeting new IMWG criteria based on the FLC assay or BMPC\% were excluded from the analysis.

Our study also found that patients with increased focal uptake but no evidence of osteolysis are also at high risk of progression to myeloma, with a $61 \%$ risk of progression within 2 years. This is similar to a report by Zamagni et al. ${ }^{15}$ who found that $12 \%$ of patients with SMM have increased focal uptake on PET-CT without underlying osteolysis. The probability of progression within 2 and 3 years for such patients $(n=9)$ was $48 \%$ and $65 \%$, respectively, in comparison with $32 \%$ and $42 \%$ for patients with a negative PET-CT $(n=64) .{ }^{15}$ Patients with increased focal uptake without underlying osteolysis should be considered to have high-risk SMM and need close observation and consideration of clinical trials testing prophylactic therapy. ${ }^{1}$ 
In summary, our study results align with the recently updated IMWG criteria for the diagnosis of MM. Patients with suspected SMM who have evidence of clear osteolytic bone destruction on PET-CT that is attributable to the underlying plasma cell disorder should be considered to have MM.

\section{CONFLICT OF INTEREST}

SK has obtained research support for clinical trials from Celgene, Millennium, Novartis, Janssen and Sanofi. AD has received research support for clinical trials from Pfizer, Jannsen, Millenium, Alnylam and Celgene. The remaining authors declare no conflict of interest. MAG has received research support from ISIS and Prothena, and honoraria from Celgene, Millennium Pharmaceuticals and Novartis. None of these are related to the article. The other authors declare no conflict of financial interests.

\section{ACKNOWLEDGEMENTS}

This work is supported, in part, by Grants CA 107476 and CA 168762 from the National Cancer Institute, Rockville, MD, USA

\section{AUTHOR CONTRIBUTIONS}

BS and SVR designed the research, analyzed the data, wrote and edited the manuscript. SK, AD, MTD, MQL, FB, DD, PK, WG and MAG participated in data interpretation, reviewed the manuscript and provided critical comments. All authors reviewed and approved the final manuscript.

\section{REFERENCES}

1 Rajkumar SV, Landgren O, Mateos MV. Smoldering multiple myeloma. Blood 2015; 125: 3069-3075.

2 Kyle RA, Remstein ED, Therneau TM, Dispenzieri A, Kurtin PJ, Hodnefield JM et al Clinical course and prognosis of smoldering (asymptomatic) multiple myeloma. $N$ Engl J Med 2007; 356: 2582-2590.

3 Rajkumar SV, Dimopoulos MA, Palumbo A, Blade J, Merlini G, Mateos M-V et al. International Myeloma Working Group Updated Criteria for the Diagnosis of Multiple Myeloma. Lancet Oncol 2014; 15: e538-e548.

4 Kyle RA, Durie BG, Rajkumar SV, Landgren O, Blade J, Merlini G et al. Monoclonal gammopathy of undetermined significance (MGUS) and smoldering (asymptomatic) multiple myeloma: IMWG consensus perspectives risk factors for progression and guidelines for monitoring and management. Leukemia 2010; 24 1121-1127.

5 Mateos MV, San Miguel JF. Treatment for high-risk smoldering myeloma. N Engl $\mathrm{J}$ Med 2013; 369: 1764-1765.
6 Perez-Persona E, Vidriales MB, Mateo G, Garcia-Sanz R, Mateos MV, de Coca AG et al. New criteria to identify risk of progression in monoclonal gammopathy of uncertain significance and smoldering multiple myeloma based on multiparameter flow cytometry analysis of bone marrow plasma cells. Blood 2007; 110: 2586-2592.

7 Kyle RA, Durie BGM, Rajkumar SV, Landgren O, Blade J, Merlini G et al. Monoclonal gammopathy of undetermined significance (MGUS) and smoldering (asymptomatic) multiple myeloma: IMWG consensus perspectives risk factors for progression and guidelines for monitoring and management. Leukemia 2010; 24: 1121-1127.

8 Larsen JT, Kumar SK, Dispenzieri A, Kyle RA, Katzmann JA, Rajkumar SV. Serum free light chain ratio as a biomarker for high-risk smoldering multiple myeloma. Leukemia 2013; 27: 941-946.

9 Dispenzieri A, Kyle RA, Katzmann JA, Therneau TM, Larson D, Benson J et al. Immunoglobulin free light chain ratio is an independent risk factor for progression of smoldering (asymptomatic) multiple myeloma. Blood 2007; 111: 785-789.

10 Bianchi G, Kyle RA, Larson DR, Witzig TE, Kumar S, Dispenzieri A et al. High levels of peripheral blood circulating plasma cells as a specific risk factor for progression of smoldering multiple myeloma. Leukemia 2013; 27: 680-685.

11 Hillengass J, Fechtner K, Weber MA, Bauerle T, Ayyaz S, Heiss C et al. Prognostic significance of focal lesions in whole-body magnetic resonance imaging in patients with asymptomatic multiple myeloma. J Clin Oncol 2010; 28: 1606-1610.

12 Merz M, Hielscher T, Wagner B, Sauer S, Shah S, Raab MS et al. Predictive value of longitudinal whole-body magnetic resonance imaging in patients with smoldering multiple myeloma. Leukemia 2014; 28: 1902-1908.

13 Rajkumar SV, Merlini G, San Miguel JF. Redefining myeloma. Nat Rev Clin Oncol 2012; 9: 494-496.

14 Regelink JC, Minnema MC, Terpos E, Kamphuis MH, Raijmakers PG, Pieters-van den Bos IC et al. Comparison of modern and conventional imaging techniques in establishing multiple myeloma-related bone disease: a systematic review. Br J Haematol 2013; 162: 50-61.

15 Zamagni E, Nanni C, Gay F, Pezzi A, Bellò M, Rambaldi R et al. The presence of FDG $\mathrm{PET} / \mathrm{CT}$ focal, not osteolytic, lesion(s) identifies a sub-group of patients with smoldering multiple myeloma with high-risk of progression into symptomatic disease. Blood (ASH Annual Meeting Abstract) 2014; 124: A3371.

This work is licensed under a Creative Commons Attribution 4.0 International License. The images or other third party material in this article are included in the article's Creative Commons license, unless indicated otherwise in the credit line; if the material is not included under the Creative Commons license, users will need to obtain permission from the license holder to reproduce the material. To view a copy of this license, visit http://creativecommons.org/licenses/ by/4.0/ 